\title{
Application of Polymerase Chain Reaction to Analysis of Antigen Receptor Rearrangements to Support Endoscopic Diagnosis of Canine Alimentary Lymphoma
}

\author{
Naoki KANEKO ${ }^{1)}$, Yoshifumi YAMAMOTO'), Yuko WADA ${ }^{1)}$, Takako SHIMOKAWA MIYAMA ${ }^{1)}$, \\ Hiroko HIRAOKA $^{1)}$, Kazuhito ITAMOTO ${ }^{2)}$, Takuya MIZUNO ${ }^{1)}$, Munekazu NAKAICHI ${ }^{3)}$, Tomoko TAKAHASHI ${ }^{4)}$, \\ Toshihiro WATARI ${ }^{5)}$ and Masaru OKUDA ${ }^{1) *}$ \\ ${ }^{1)}$ Laboratories of Veterinary Internal Medicine, ${ }^{2)}$ Veterinary Surgery and ${ }^{3)}$ Veterinary Radiology, Faculty of Agriculture, Yamaguchi \\ University, 1677-1 Yoshida, Yamaguchi-shi, Yamaguchi 753-8515 and ${ }^{4}$ Laboratories of Veterinary Radiology and \\ ${ }^{5)}$ Comprehensive Veterinary Clinical Studies, Department of Veterinary Medicine, College of Bioresource Sciences, Nihon University, \\ 1866 Kameino, Fujisawa-shi, Kanagawa 252-8510, Japan
}

(Received 7 November 2008/Accepted 21 November 2008)

ABSTRACT. We evaluated the usefulness of polymerase chain reaction for antigen receptor gene rearrangement analysis (PARR) of endoscopic biopsy specimens for diagnosis of canine alimentary lymphoma. Two endoscopic biopsy specimens were obtained from each lesion in 78 dogs with gastrointestinal symptoms. One specimen was histopathologically examined by a pathologist, and the other was analyzed by PARR. All samples were categorized into three groups [lymphoma $(n=4)$, adenocarcinoma $(n=5)$ and enteritis groups $(n=69)]$ based on the histopathological diagnosis. In the lymphoma group, one case was IgH major-positive, and three cases were TCR $\gamma$ positive, representing clonal expansion of B- and T-cells, respectively. PARR produced negative results for all cases in the adenocarcinoma group. In the enteritis group, six cases were TCR $\gamma$-positive. Two of the six TCR $\gamma$-positive enteritis cases were cytologically diagnosed as lymphoma by fine needle aspiration during a laparotomy. In the enteritis group, the survival times were compared between the TCR $\gamma$-positive and TCR $\gamma$-negative cases. The overall survival time of the TCR $\gamma$-positive enteritis cases was significantly shorter than that of the TCR $\gamma$-negative enteritis cases according to a log-rank test $(p<0.0001)$. With regard to other factors, such as age, clinical signs and the serum albumin concentration, there were no significant differences between the TCR $\gamma$-positive and TCR $\gamma$-negative enteritis cases. In conclusion, PARR is capable of detecting alimentary lymphoma and latent alimentary lymphoma, which cannot be histopathologically diagnosed using endoscopic biopsy specimens. Furthermore, a TCR $\gamma$-positive result in PARR may imply a poor prognosis. KEY WORDS: diagnosis, enteric disease, IBD, Lymphoma/leukemia, molecular identification.

J. Vet. Med. Sci. 71(5): 555-559, 2009

Lymphoma is one of the most common tumors in dogs. Alimentary lymphoma usually accounts for $5 \%$ to $7 \%$ of all canine lymphomas [16]. Dogs with alimentary lymphoma show nonspecific gastrointestinal signs, such as vomiting, diarrhea, anorexia, weight loss, hypoproteinemia and malabsorption. Alimentary lymphoma usually occurs multifocally and/or diffusely throughout the submucosa and lamina propria of the intestine, especially in the small intestine, with frequent superficial ulceration and occasional transmural infiltration of the serosa [18]. Although alimentary lymphomas that involve formation of a mass can be detected by diagnostic imaging, it is difficult to detect diffuse alimentary lymphomas that do not involve a mass. Lymphocyticplasmacytic inflammation can occur adjacent to or distant from the primary tumor, and distinguishing between alimentary lymphoma and enteritis, especially lymphocytic-plasmacytic enteritis (LPE), can be difficult [4, 18]. Additionally, interobserver variation is also an issue in relation to histopathologic evaluation of intestinal tissues [19]. Therefore, full thickness biopsy of the intestine is helpful in making an accurate diagnosis; however, dogs that are suspected of having alimentary lymphoma are usually in poor

* Correspondence to: OKudA, M., Laboratory of Veterinary Internal Medicine, Faculty of Agriculture, Yamaguchi University, 1677-1 Yoshida, Yamaguchi 753-8515, Japan.

e-mail: okudamu@yamaguchi-u.ac.jp condition and are unlikely to survive a long period of anesthesia, and laparotomic damage may also occur in some cases. There is also a risk of intestinal perforations at the laparotomic biopsy site in dogs with hypoalbuminemia or intensive infiltration of inflammatory cells and/or lymphoma cells into the intestine [13]. In contrast, endoscopic biopsy is less invasive than laparotomy, but is considered to be inadequate because only small, superficial (mucosa) specimens can be obtained $[9,18]$.

The polymerase chain reaction (PCR) for antigen receptor gene rearrangement analysis (PARR) was developed for diagnosis of lymphoid neoplasia [3, 17]. Lymphocytes have acquired a unique antigen receptor gene of a unique length and sequence by gene rearrangement. The lymphoid neoplasia is composed of a single population of neoplastic lymphocytes. In PARR, clonal expansion of tumor lymphocytes that contain a rearranged immunoglobulin heavy chain (IgH) gene or a T cell receptor gamma (TCR $\gamma)$ gene can be detected in B-cell and T-cell lymphoid neoplasias, respectively. PARR is an objective and highly sensitive method, and its usefulness in diagnosis of canine lymphoid neoplasia has recently been proven [3, 8, 10, 17]. In the present study, we evaluated the usefulness of PARR using endoscopic biopsy specimens in diagnosis of alimentary lymphoma. 


\section{MATERIALS AND METHODS}

Tissue specimens: The present study utilized 78 dogs that suffered from gastrointestinal symptoms such as diarrhea and vomiting and that had been examined by endoscopy at the Yamaguchi University Animal Medical Center, Nihon University Animal Medical Center and five other private animal hospitals between October 2003 and October 2005. Two endoscopic biopsy specimens were obtained from each lesion with endoscopic biopsy forceps. One of the specimens was fixed in $10 \%$ buffered formalin and evaluated histopathologically in each hospital's pathological laboratories or by commercial laboratories. All the specimens were classified into three groups (lymphoma, adenocarcinoma and enteritis groups) based on the histopathological diagnosis. The other specimen was placed in $200 \mu l$ of saline and stored at $-20^{\circ} \mathrm{C}$ until use.

PARR: Each endoscopic biopsy specimen was homogenized, and DNA was extracted using QIAamp DNA Mini Kits (QIAGEN GmbH, Hilden, Germany) according to the manufacturer's instructions. PARR was performed as described previously [3]. Briefly, each sample was amplified by two sets of primers (major and minor) for the IgH gene and one set of primers for the TCR $\gamma$ gene. As a positive control for the DNA extraction process, the constant region of $\operatorname{IgM}(\mathrm{C} \mu)$ was also amplified for each sample. The PCR products were electrophoresed using $12 \%$ polyacrylamide gel, and the gel was stained with ethidium bromide and visualized with an UV illuminator. If one or more distinct bands were observed, the results were considered to be positive, i.e., one or more tumor lymphocyte clonal expansions were present in the sample. If no bands, some smears or a ladder of faint bands were observed, the results were considered to be negative. If tissue specimens were obtained from more than two lesions in a dog, the PARR results for a pathologically representative lesion were considered to be the clonality results for that dog in the statistical analysis.

Statistical analysis: In the enteritis group, the overall survival time, age and serum albumin concentration were compared between the TCR $\gamma$-positive and TCR $\gamma$-negative cases. Overall survival time was evaluated using the Kaplan-Meier method and the log-rank test. The age and albumin concentration were examined for normality using the D'Agostino and Pearson normality test and were compared using the Mann-Whitney $U$ test. The clinical signs were compared using the Fisher's exact test. A $p$ value $<0.05$ was considered statistically significant. The calculations were performed using statistical software (Prism 5, GraphPad Software Inc., San Diego, CA, U.S.A.).

\section{RESULTS}

Dogs: The median age of the 78 dogs was 6.0 years (range: seven months to 16 years), and their median weight was $6.8 \mathrm{~kg}$ (range: 2.3 to $53 \mathrm{~kg}$ ). Forty-seven dogs were male (31 intact, 16 neutered), and 31 dogs were female (14 intact, 17 neutered). Seventy dogs representing 27 pure breeds were affected, as were eight dogs of mixed breed. The most commonly affected breeds were the Miniature Dachshund ( $n=14)$, Shih Tzu $(n=5)$, West Highland White Terrier $(n=4)$, Pembroke Welsh Corgi $(n=4)$ and Shiba $(n=4)$. The clinical signs observed were vomiting $(n=50)$, diarrhea $(n=40)$, anorexia $(n=32)$, lethargy $(n=24)$ and effusion $(n=4)$. The mean ( \pm standard deviation) albumin concentration of 68 of the dogs for the seven days before the endoscopy was $2.5 \pm 0.9 \mathrm{~g} / \mathrm{d} \mathrm{l}$.

Histopathological diagnosis: The endoscopic specimens were examined histopathologically by pathologists. The pathologists diagnosed cases of lymphoma $(n=2)$; cases suggestive of lymphoma $(\mathrm{n}=2)$; and cases of adenocarcinoma $(n=5)$, LPE $(n=27)$, chronic enteritis $(n=39)$, haemorrhagic enteritis $(n=2)$ and polyp $(n=1)$. These cases were classified into three groups: the lymphoma, adenocarcinoma and enteritis groups. The lymphoma group included the lymphoma and suggestive of lymphoma cases. The adenocarcinoma group included only the adenocarcinoma cases. The enteritis group included the LPE, chronic enteritis, haemorrhagic enteritis and polyp cases.

PARR: Based on the histopathological observations, specimens obtained from the stomach $(n=8)$, duodenum $(n=65)$ and colon $(n=5)$ were considered to represent the main lesions in each dog, and the PARR results for the main lesion were considered to represent the clonality results for each case. In PARR, $\mathrm{C} \mu$ was successfully amplified as a control for the DNA extraction process in all cases, and this confirmed successful DNA extraction for the specimens. IgH major was positive in one lymphoma case. TCR $\gamma$ was positive in three lymphoma cases and six enteritis cases. IgH minor was negative in all cases, and there were no multi-positive cases. Therefore, all the lymphoma cases were positive according to PARR. Importantly, two of the six TCR $\gamma$-positive enteritis cases, which were not diagnosed as lymphoma based on the endoscopic specimens, were cytologically diagnosed as lymphoma by fine needle aspiration during a laparotomy. In the other four TCR $\gamma$-positive enteritis cases, neither laparotomy nor autopsy was performed. All the adenocarcinoma cases produced negative PARR results.

Comparison of histopathology and PARR: Follow-up information was available in 55 cases. All the lymphoma cases and the two enteritis cases that had been cytologically diagnosed as lymphoma were treated with multidrug (CHOP-based protocol) and/or lomustine chemotherapy. One TCR $\gamma$-positive enteritis case, which was not diagnosed as lymphoma by histopathology, was also treated with multidrug chemotherapy, but this dog died 19 days after endoscopy. The other enteritis cases were treated with a modified diet, metronidazole, sulfasalazine and/or immunosuppressive therapy, including prednisolone, azathioprine and cyclosporine.

The overall survival time of the TCR $\gamma$-positive enteritis cases was compared with that of the TCR $\gamma$-negative enteritis cases. Follow-up information was available in six TCR $\gamma$ positive and 45 TCR $\gamma$-negative enteritis cases. The overall 


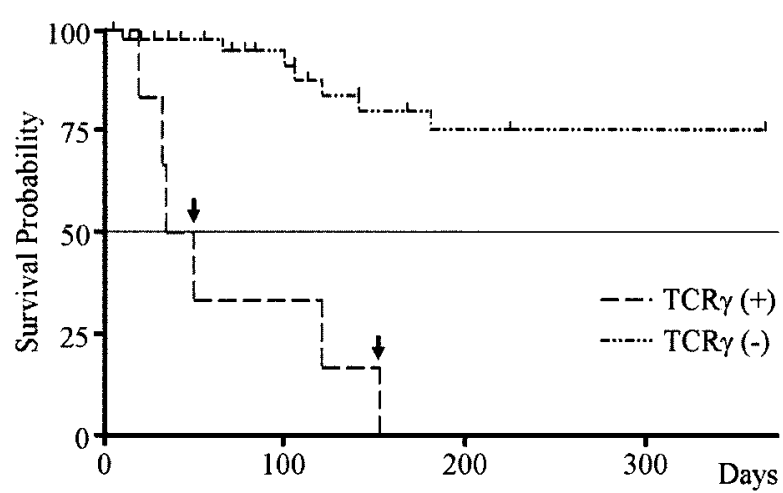

Fig. 1. Kaplan-Meier curve for the survival times of the TCR $\gamma$ positive and TCR $\gamma$-negative cases in the enteritis group. There were six and 45 TCR $\gamma$-positive $(+)$ and TCR $\gamma$-negative (-) cases, respectively. The arrows indicate the survival times of the TCR $\gamma(+)$ cases cytologically diagnosed as lymphoma during laparotomy. Censored cases, which involved death by other diseases or which could not be followed up subsequently, are indicated by tick marks.

survival time of the TCR $\gamma$-positive enteritis cases was significantly shorter than that of the TCR $\gamma$-negative enteritis cases according to the log-rank test $(p<0.0001$; Fig. 1$)$. The 2 lymphoma cases in the enteritis group, which had been cytologically diagnosed as lymphoma at the laparotomy, died at 34 and 153 days, respectively (Fig. 1). With regard to the other conditions, including age, clinical symptoms and serum albumin concentration, there were no significant differences between the TCR $\gamma$-positive and TCR $\gamma$-negative enteritis cases (Table 1).

\section{DISCUSSION}

In all the cases in the lymphoma group, the clonalities of the antigen receptor genes were detected with PARR using endoscopic biopsy specimens. The sensitivity of PARR for histopathologic diagnosis was $100 \%$, although the number of samples was limited. PARR has been shown to be a highly sensitive method, but it sometimes produces false negative results if the clonality is not detected with the primers used, if the lymphoid neoplasia originates from natural killer cells or if the malignant cells are present in numbers too low to be detected [1]. All the adenocarcinoma cases in the present study produced negative PARR results. This confirms that PARR does not detect tumors other than lymphoid neoplasias, even if the tumors possess tumor cells containing the clonal gene, because the antigen receptor genes of these other tumors are not rearranged.

The clonality of TCR $\gamma$ was detected in six enteritis cases. Lymphoma was diagnosed cytologically during laparotomy in two of these six cases. This suggests that PARR can detect latent lymphoma cases that cannot be histopathologically diagnosed as lymphoma using endoscopic biopsy specimens. In the present study, the pathologists did not identify the specimens as lymphoma. This may have resulted from subtle differences between the biopsy specimens because they were taken from close, but not identical lesions. Moreover, it has been reported that interobserver variation and the quality of endoscopic biopsy specimens influences histopathological outcome $[19,20]$. Since the endoscopic biopsy specimens were taken by several operators and evaluated by several pathologists, this may have influenced the histopathological diagnoses obtained in the present study. Recently, The World Small Animal Veterinary Association Gastrointestinal Standardization Group standardized the method for diagnosis of gastrointestinal inflammation in endoscopic biopsy specimens from dogs and cats [6]. It is important to use this protocol in future studies.

Lymphoma was not diagnosed in the remaining four cases. It is unknown whether they were true lymphomas because they were not examined by laparotomy or autopsy. Recently, we experienced a canine case that was histopathologically diagnosed as LPE according to an endoscopic biopsy specimen but was TCR $\gamma$-positive according to PARR. This dog did not respond to treatment (metronidazole, prednisolone and azathioprine) and was confirmed as having LPE by autopsy (Kaneko and Okuda, unpublished observations). False positive PARR results have been reported for some infectious diseases, such as ehrlichiosis [3] and Lyme disease. Therefore, it is possible that the PARR results of the four TCR $\gamma$-positive enteritis cases were false positives. In order to clarify this, we cloned the PCRamplified products into a plasmid vector and performed

Table 1. Comparison of the TCR $\gamma$-positive and TCR $\gamma$-negative cases in the enteritis group

\begin{tabular}{lccccc}
\hline & TCR $\gamma(+)$ & $\mathrm{n}^{\text {b) }}$ & TCR $\gamma(-)$ & $\mathrm{n}^{\text {b) }}$ & $p$ value \\
\hline Age ${ }^{\text {a) }}$ (years $)$ & $8.3 \pm 3.7$ & 6 & $6.0 \pm 3.4$ & 63 & 0.157 \\
Clinical signs & & & & & \\
$\quad$ Vomiting & 5 & 6 & 38 & 63 & 0.398 \\
$\quad$ Diarrhea & 4 & 6 & 32 & 63 & 0.675 \\
Anorexia & 3 & 6 & 25 & 63 & 0.681 \\
Lethargy & 1 & 6 & 20 & 63 & 0.659 \\
Effusion & 0 & 6 & 4 & 63 & 1.00 \\
Weight Loss & 1 & 4 & 5 & 37 & 0.483 \\
Albumin $^{\text {a) }}(\mathrm{g} / \mathrm{d} l)$ & $1.9 \pm 0.3$ & 5 & $2.5 \pm 0.9$ & 57 & 0.07 \\
\hline
\end{tabular}

a) Continuous data is reported as the mean \pm standard deviation.

b) The number of cases whose data was complete. 
sequence analysis. However, we could not obtain their sequences, probably due to their short lengths. We recently showed that application of single-strand conformation polymorphism analysis (SSCP) for PARR provides clearer results than conventional PARR because SSCP separates different DNA sequences based on their single-strand secondary structure conformations, even if they are the same length [7]. Use of both PARR and SSCP (PARR-SSCP analysis) instead of sequence analysis may help to clarify this point in the future.

In the enteritis group, the overall survival times were compared between the TCR $\gamma$-positive and TCR $\gamma$-negative cases. The survival time of the TCR $\gamma$-positive enteritis cases was significantly shorter than that of TCR $\gamma$-negative enteritis cases $(p<0.0001$; Fig. 1$)$. The survival durations of the two TCR $\gamma$-positive cases diagnosed as lymphoma by laparotomy were not shorter than the other cases (Fig. 1), suggesting that the poor survival time was not attributed to the two lymphoma cases. This result suggests that a TCR $\gamma$ positive result in enteritis cases implies a poor prognosis. Other factors were also compared between the TCR $\gamma$-positive and TCR $\gamma$-negative enteritis cases (Table 1). Age, clinical symptoms and serum albumin concentration were not significantly associated with TCR $\gamma$ clonality, although some parameters, including serum albumin concentration and weight loss, have been reported to be prognostic factors of inflammatory bowel disease and LPE [5, 12]. These findings suggest that TCR $\gamma$ clonality was the only negative factor for survival time in the enteritis group. It has been reported that the neoplastic lymphocytes of the canine alimentary lymphoma usually originate from $\mathrm{T}$ cells [11] and that canine T-cell lymphoma cases are associated with shorter survival durations [14, 15]. However, the PARR results of the four TCR $\gamma$-positive enteritis cases could have been false positives as described above. We could not elucidate why TCR $\gamma$-false positive enteritis cases would have a poor prognosis (if this is the case).

LPE can occur adjacent to or distant from the primary tumor $[4,18]$. In addition, it is difficult to diagnosis lymphoma histopathologically when only a small number of malignant lymphocytes are present among the normal lymphocytes and plasma cells. In contrast, PARR is able to detect the clonality when $0.1-10 \%$ of the DNA is derived from neoplastic cells [3]. Furthermore, LPE has been described in basenjis that subsequently developed alimentary lymphoma [2]. Therefore, the TCR $\gamma$-positive results in the enteritis cases may imply that PARR is capable of detecting latent lymphoma or prelymphomatous changes in endoscopic biopsy specimens. If a clonal TCR $\gamma$ gene is detected in endoscopic biopsy specimens, even if the specimens have been histopathologically diagnosed as enteritis, additional examinations, including laparotomy or laparoscopy, are recommended to search for latent alimentary lymphoma.

In conclusion, in the present study PARR was able to detect alimentary lymphoma and latent alimentary lymphoma, which cannot be diagnosed histopathologically using an endoscopic biopsy. Furthermore, the results suggest that the presence of a clonal TCR $\gamma$ gene implies a poor prognosis and a lymphoma or prelymphomatous change in dogs suffering from enteritis. However, due to the small numbers of lymphoma- and PARR-positive cases, the lack of pathological standardization and the lack of uniformity in treatment, further studies are necessary to confirm the relationship between TCR $\gamma$ clonality and prognosis.

ACKNOWLEDGEMENTS. This research was supported by a grant-in-aid from the Ministry of Education, Culture, Sports, Science and Technology of Japan (15380218). The authors would like to thank the following cooperating hospitals: Nihon University Animal Medical Center, Azabu University Veterinary Teaching Hospital, Sanyo Animal Medical Center, Takahashi Pet Clinic and Nakamura Animal Hospital.

\section{REFERENCES}

1. Avery, P. R. and Avery, A. C. 2004. Molecular methods to distinguish reactive and neoplastic lymphocyte expansions and their importance in transitional neoplastic states. Vet. Clin. Pathol. 33: 196-207.

2. Breitschwerdt, E. B., Waltman, C., Hagstad, H. V., Ochoa, R., McClure, J. and Barta, O. 1982. Clinical and epidemiological characterization of a diarrheal syndrome in Basenji dog. J. Am. Vet. Med. Assoc. 180: 914-920.

3. Burnett, R. C., Vernau, W., Modiano, J. F., Olver, C. S., Moore, P. F. and Avery, A. C. 2003. Diagnosis of canine lymphoid neoplasia using clonal rearrangements of antigen receptor genes. Vet. Pathol. 40: 32-41.

4. Couto, C. G., Rutgers, H. C., Sherding, R.G. and Rojko, J. 1989. Gastrointestinal lymphoma in 20 dogs. A retrospective study. J. Vet. Intern. Med. 3: 73-78.

5. Craven, M., Simpson, J. W., Ridyard, A. E. and Chandler, M. L. 2004. Canine inflammatory bowel disease: retrospective analysis of diagnosis and outcome in 80 cases (1995-2002). J. Small Anim. Pract. 45:336-342.

6. Day, M. J., Bilzer, T., Mansell, J., Wilcock, B., Hall, E. J., Jergens, A., Minami, T., Willard, M. and Washabau, R.; World Small Animal Veterinary Association Gastrointestinal Standardization Group. 2008. Histopathological standards for the diagnosis of gastrointestinal inflammation in endoscopic biopsy samples from the dog and cat: a report from the World Small Animal Veterinary Association Gastrointestinal Standardization Group. J. Comp. Pathol. 138: S1-43.

7. Kaneko, N., Tanimoto, T., Morimoto, M., Hayashi, T., Miyama Shimokawa, T., Hiraoka, H., Itamoto, K., Une, S., Mizuno, T. and Okuda, M. The use of formalin-fixed paraffinembedded tissue and single-stranded conformation polymorphism analysis for polymerase chain reaction of antigen receptor rearrangements in dogs. J. Vet. Med. Sci. (in press).

8. Keller, R. L., Avery, A. C., Burnett, R. C., Walton, J. A. and Olver, C. S. 2004. Detection of neoplastic lymphocytes in peripheral blood of dogs with lymphoma by polymerase chain reaction for antigen receptor gene rearrangement. Vet. Clin. Pathol. 33: 145-149.

9. Kleinschmidt, S., Meneses, F., Nolte, I. and Hewicker-Trautwein, M. 2006. Retrospective study on the diagnostic value of full-thickness biopsies from the stomach and intestines of dogs 
with chronic gastrointestinal disease symptoms. Vet. Pathol. 43: 1000-1003.

10. Lana, S. E., Jackson, T. L., Burnett, R. C., Morley, P. S. and Avery, A. C. 2006. Utility of polymerase chain reaction for analysis of antigen receptor rearrangement in staging and predicting prognosis in dogs with lymphoma. J. Vet. Intern. Med. 20: 329-334.

11. Miura, T., Maruyama, H., Sakai, M., Takahashi, T., Koie, H., Yamaya, Y., Shibuya, H., Sato, T., Watari, T., Tokuriki, M. and Hasegawa, A. 2004. Endoscopic findings on alimentary lymphoma in 7 dogs. J. Vet. Med. Sci. 66: 577-580.

12. Ohno, K., Konishi, S., Kobayashi, S., Nakashima, K., Setoguchi, A., Fujino, Y., Nakayama, H. and Tsujimoto, H. 2006. Prognostic factors associated with survival in dogs with lymphocytic-plasmacytic enteritis. J. Vet. Med. Sci. 68: 929-933.

13. Ralphs, S. C., Jessen, C. R. and Lipowitz, A. J. 2003. Risk factors for leakage following intestinal anastomosis in dogs and cats: 115 cases (1991-2000). J. Am. Vet. Med. Assoc. 223: 7377.

14. Ruslander, D. A., Gebhard, D. H., Tompkins, M. B., Grindem, C. B. and Page, R. L. 1997. Immunophenotypic characterization of canine lymphoproliferative disorders. In Vivo. 11: 169172.

15. Teske, E., van Heerde, P., Rutteman, G. R., Kurzman, I. D.,
Moore, P. F. and MacEwen, E. G. 1994. Prognostic factors for treatment of malignant lymphoma in dogs. J. Am. Vet. Med. Assoc. 205: 1722-1728.

16. Theilen, G. H. and Madewell, B. R. 1987. pp. 392-407. In: Veterinary Cancer Medicine, 2nd ed. (Theilen, G. H. and Madewell, B. R. eds.), Lea and Febiger, Philadelphia.

17. Valli, V. E., Vernau, W., de Lorimier, L. P., Graham, P. S. and Moore, P. F. 2006. Canine indolent nodular lymphoma. Vet. Pathol. 43: 241-256.

18. Vail, D.M. and Young, K.M. 2007. Canine lymphoma and lymphoid leukemias. pp. 600-733. In: Small Animal Clinical Oncology, 4th ed. (Withrow, S. J. and Vail, D. M. eds.), Saunders, Philadelphia.

19. Willard, M. D., Jergens, A. E., Duncan, R. B., Leib, M. S., McCracken, M. D., DeNovo, R. C., Helman, R. G., Slater, M. R. and Harbison, J. L. 2002. Interobserver variation among histopathologic evaluations of intestinal tissues from dogs and cats. J. Am. Vet. Med. Assoc. 220: 1177-1182.

20. Willard, M. D., Mansell, J., Fosgate, G. T., Gualtieri, M., Olivero, D., Lecoindre, P., Twedt, D. C., Collett, M. G., Day, M. J., Hall, E. J., Jergens, A. E., Simpson, J. W., Else, R. W. and Washabau, R. J. 2008. Effect of sample quality on the sensitivity of endoscopic biopsy for detecting gastric and duodenal lesions in dogs and cats. J. Vet. Intern. Med. 22: 1084-1089. 